\title{
A NOTE ON THE RELATIONSHIP BETWEEN WEIL AND CARTIER DIVISORS
}

\author{
JAMES HORNELL
}

ABSTRACT. Using a generalized equivalence relation, a subquotient of the group of Weil divisors is shown to be isomorphic to the group of Cartier divisors modulo linear equivalence for a reduced subscheme of a projective space over a field. A difficulty of the nonreduced case is discussed.

Let $X, \Theta$ be a subscheme of a projective space over a field. A generalized equivalence relation is defined on the group of Weil divisors, and if $X$, $\mathcal{O}$ is reduced, a corresponding subquotient is shown to be isomorphic to the group of Cartier divisors modulo linear equivalence. The generalized equivalence for curves appears in [5]. Projectivity may be replaced by the conditions that any finite number of points of $X$ lie in an affine open of $X$ and that the nonregular locus of $X, \mathcal{O}$ is closed. An example is given which shows one difficulty of the nonreduced case.

By a prime ideal $p$ of $\mathcal{O}$ is meant a subsheaf $p$ of ideals of $\mathcal{O}$ such that for every open $U \subset X, \Gamma(U, p)$ is a prime ideal of $\Gamma(U, \mathcal{O})$. These are the points of $X$ by the usual correspondence.

For reducible schemes, the notation for zero-divisors developed in [2] is used. A zero prime ideal $N$ of $\Theta$ is a proper prime ideal of $\mathcal{O}$ such that for each open $U \subset X, \Gamma(U, N)$ is either $\Gamma(U, \mathcal{O})$ or consists entirely of zero divisors of $\Gamma(U, \mathcal{O})$. A divisorial prime ideal $p$ of $\mathcal{O}$ is a prime ideal $p$ of $\mathcal{O}$ which contains a zero prime ideal $N$ of $\Theta$ such that $p / N$ is of height one in $\Theta / N$.

Let $Q \mathcal{O}$ denote the total quotient sheaf of $\Theta$, and let $K=\Gamma(X, Q \Theta)$. Let $\mathscr{O}=\Gamma\left(X,(Q \mathcal{O})^{*} / \mathcal{O}^{*}\right)$, the group of Cartier divisors, let $\Re=$ $\Gamma\left(X,(Q \mathcal{O})^{*}\right) / \Gamma\left(X, \mathcal{O}^{*}\right)$, and let $(\mathfrak{S}=\mathcal{D} / \mathfrak{\beta}$. ( $\mathbb{\beta}$ is the set of principal Cartier divisors, and $\Re$ defines linear equivalence on $D)$ ) Let $(f)$ denote the principal Cartier divis or defined by $f \in K^{*}$.

Received by the editors January 31, 1973 and, in revised form, May 3, 1974. AMS (MOS) subject classifications (1970). Primary 14C20; Secondary 14B05, $14 \mathrm{C} 10$.

Key words and phrases. Divisors, Cartier divisors, generalized equivalence relation. 
Let $\mathscr{D} \subset X$ be the set of nondivisorial prime ideals $p$ of $\mathcal{O}$ such that if $f$ is a regular element of $\Theta_{p}$ then $\Theta_{p} p$ is an embedded prime ideal of $\Theta_{p} f$. Let $\mathcal{C}$ be the union of $\mathfrak{D}$ and the set of nonnormal (nonregular) divisorial prime ideals. If $X, \mathcal{O}$ is reduced, then $\mathcal{D}$ is the set of nondivisorial prime ideals of depth (grade) one, and both $\mathcal{D}$ and $\mathcal{C}$ are finite.

Let $D$ be the group of Weil divisors of $X$, the free abelian group generated by the divisorial prime ideals of $\Theta$. Let $p$ be a divisorial prime ideal of $\mathcal{O}$, let $f \in K^{*}$, let $f_{1}$ and $f_{2}$ be regular elements of $\mathcal{O}_{p}$ such that $f_{p}=$ $f_{1} / f_{2}$, and define

$$
\lambda_{p}(f)=\sum_{N \subset p}\left(\rho_{\Theta_{p}}\left(\frac{\Theta_{p}}{\mathcal{O}_{p} f_{1}+N_{p}}\right)-\rho_{\Theta_{p}}\left(\frac{\Theta_{p}}{\hat{O}_{p} f_{2}+N_{p}}\right)\right),
$$

where the sum is over all zero prime ideals $N \subset p$ with $p / N$ of height one in $\Theta / N$. Let the principal Weil divisor defined by $f \in K^{*}$ be $\langle f\rangle=\Sigma_{p} \lambda_{p}(f) p$, where the sum is over all divisorial prime ideals $p$. Let $S$ be the set of all divisorial prime ideals which are contained in some element of $\mathcal{C}$. Let $D \backslash S$ be the free abelian group generated by those divisorial prime ideals not be. longing to $S$. Let $I$ be the subgroup of $D$ of principal divisors $\langle f\rangle$ where $f \in K^{*}$ is such that $f_{q} \in \mathcal{O}_{q}^{*}$ for all $q \in \mathcal{C}$, consider $I$ as a subgroup of $D \backslash S$, and let $C=(D \backslash S) / I$. (D/I is the generalized class group of $X, \mathcal{O}$ and $I$ de. fines a generalized equivalence relation. Letting $P$ be the subgroup of $D$ of all principal divis ors $\langle f\rangle, D / P$ is the class group of $X, \mathcal{O}$, and $P$ defines linear equivalence on $D$. )

To construct the injection from $(5)$ to $C$ for $X, O$ reduced, only the follow ing approximation lemma for Cartier divisors is needed. It is a variant of the usual approximation [3, Lemma 3, p. 166].

Recall that if $p_{1}, \cdots, p_{s}$ and $p$ are prime ideals of $\mathcal{O}$, then $p \subset p_{1} \cup \cdots$ $\cup p_{s}$ if and only if $p \subset p_{i}$ for some $i=1, \cdots, s$. Let $q_{1}, \cdots, q_{t}$ also be prime ideals of $\mathcal{O}$. The condition below that

$$
p \subset\left(p_{1} \cup \cdots \cup p_{s}\right) \cap\left(q_{1} \cup \cdots \cup q_{t}\right)
$$

is equivalent to $p \subset p_{i} \cap q_{j}$ for some $i$ and $j$.

Lemma. Let $X, \mathcal{O}$ be a subscheme of a projective space over a field. Let $p_{1}, \cdots, p_{s}$ be prime ideals of $\mathcal{O}$, let. $D$ be a Cartier divisor on $X$, and let $q_{1}, \cdots, q_{t}$ be prime ideals of $\mathcal{E}$ such that $D_{p}=(1)_{p}$ for every prime ideal $p$ of $\mathcal{O}$ with $p \subset\left(p_{1} \cup \cdots \cup p_{s}\right) \cap\left(q_{1} \cup \cdots \cup q_{t}\right)$. Then there is an 
element $f$ of $K^{*}$ such that $(f)_{p_{i}}=D_{p_{i}}$ for $i=1, \cdots, s$ and $(f)_{q_{i}}=(1)_{q_{i}}$ for $i=1, \cdots, t$.

Proof. First assume the Lemma is true for $s=1$, and induct on $s$. Assuming true for $s-1$, there is an $f^{\prime \prime} \in K^{*}$ with $\left(f^{\prime \prime}\right)_{q_{i}}=(1) q_{q_{i}}$ for $i=1, \cdots, t$ and $\left(f^{\prime \prime}\right)_{p_{i}}=D_{p_{i}}$ for $i=1, \cdots, s-1$. Then $\left(D-\left(f^{\prime \prime}\right)\right)_{p_{i}}=(1)_{p_{i}}$ for $i=1, \cdots$, $s-1$, and $\left(D-\left(f^{\prime \prime}\right)\right)_{q_{i}}=D_{q_{i}}$ for $i=1, \cdots, t$. It follows that if $p$ is a prime ideal with

$$
p \subset\left(p_{1} \cup \cdots \cup p_{s-1} \cup q_{1} \cup \cdots \cup q_{t}\right) \cap p_{s}
$$

then $\left(D-\left(f^{\prime \prime}\right)\right)_{p}=(1)_{p}$. Thus there is an $f^{\prime} \in K^{*}$ such that $\left(f^{\prime}\right)_{p_{s}}=$ $\left(D-\left(f^{\prime \prime}\right)\right)_{p_{s}},\left(f^{\prime}\right)_{p_{i}}=(1)_{p_{i}}$ for $i=1, \cdots, s-1$, and $\left(f^{\prime}\right)_{q_{i}}=(1) q_{q_{i}}$ for $i=1, \cdots$, t. Let $f=f^{\prime \prime} / f^{\prime} \in K^{*}$.

Now let $s=1$ and $p=p_{1}$. Let $A=\Gamma(U, \mathcal{O})$ where $U$ is an affine open subset of $X$ meeting each irreducible component of $X$ and containing the points $q_{1}, \cdots, q_{t}$ and $p$ which are to be considered as prime ideals of $A$. Let $g_{1}$ and $g_{2}$ be regular elements of $A$ such that $D_{p}=\left(g_{1} / g_{2}\right)_{p}$.

Let $P_{1}, \cdots, P_{r}$ be the associated prime ideals of $A g_{2}$ for which $g_{1} / g_{2}$ $\notin A_{P_{i}} \cdot P_{i}$ is not a zero prime ideal because $g_{2} \in P_{i}$. If $P_{i} \subset p$ then $D_{P_{i}}=$ $\left(g_{1} / g_{2}\right)_{P_{i}} \not(1)_{P_{i}}$ and $P_{i} \not \subset q_{1} \cup \cdots \cup q_{t}$, and let $e_{i} \in P_{i}$ be a regular element of $A$ such that $e_{i} \notin q_{1} \cup \cdots \cup q_{t}$. Or, if $P_{i} \not \subset p$, let $e_{i} \in P_{i}$ be a regular element of $A$ with $e_{i} \notin p$. There is an integer $n \geq 1$ such that $\left(e_{1} \cdots e_{r}\right)^{n} g_{1} \epsilon$ $A g_{2}$. Let $h_{1}=\left(e_{1} \cdots e_{r}\right)^{n} g_{1} g_{2}^{-1} \in A$, and let $h_{2}=\left(e_{1} \cdots e_{r}\right)^{n}$. Then $D_{p}=$ $\left(b_{1} / h_{2}\right)_{p}$, and no isolated prime ideal of $A h_{1}$ or $A h_{2}$ is contained in $p \cap$ $\left(q_{1} \cup \cdots \cup q_{t}\right)$.

Let $Q_{1}, \cdots, Q_{s}$ be the associated prime ideals of $A 0$ in $A$. For $j=1$, 2 , let $k_{j}$ be an element of $p$ contained in all the isolated prime ideals of $A_{p} h_{j}$ such that for $i=1, \cdots, t, k_{j} \in q_{i}$ if and only if $b_{j} \notin q_{i}$, and such that for $i=1, \cdots, r, k_{j} \in Q_{i}$ if $Q_{i} \not \subset q_{1} \cup \cdots \cup q_{t}$. There is an integer $n \geq 1$ such that $k_{j}^{n} \in A_{p} h_{j}$ for $j=1,2$, for $k_{j}$ is contained in each associated prime ideal of $A_{p} h_{j}$. For $j=1,2$, let $f_{j}=h_{j}+k_{j}^{n+1}$. Then $f_{j} \notin q_{1} \cup \ldots \cup q_{t}, f_{j}$ is a regular element of $A, 1+b_{j}^{-1} k_{j}^{n+1}$ is a unit of $A_{p}$, and $\left(f_{1} / f_{2}\right)_{p}=D_{p}$. Let $f=f_{1} / f_{2}$. Q.E.D.

The injection $\phi: \mathbb{S} \rightarrow C$ is to be constructed. Let $\alpha \in \mathbb{S}$. By the Lemma there is a divisor $D$ in $\alpha$ such that $D_{p}=(1)_{p}$ for all $p \in \mathcal{C}$, and therefore $D_{p}=$ 
(1) for all $p \in S$. For a divisorial prime ideal $p$, let $f_{1}$ and $f_{2}$ be regular elements of $\mathcal{O}_{p}$ with $D_{p}=\left(f_{1} / f_{2}\right)_{p}$, and define

$$
\lambda_{p}(D)=\sum_{N \subset \theta_{p}}\left(\rho_{\theta_{p}}\left(\frac{\Theta_{p}}{\mathcal{O}_{p} f_{1}+N}\right)-\ell_{\theta_{p}}\left(\frac{\Theta_{p}}{\hat{\Theta}_{p} f_{2}+N}\right)\right),
$$

where the sum is over all zero prime ideals $N$ of $\mathcal{O}_{p}$ such that $\mathcal{O}_{p} p / N$ is of he ight one in $\mathcal{O}_{p} / N$. Let

$$
\lambda D=\sum_{p \notin S} \lambda_{p}(D) p \in D \backslash S,
$$

where the sum is over all divisorial prime ideals $p$ not contained in $S$. If $f \in K^{*}$ and $(f)_{p}=(1)_{p}$ for all $p \in \mathcal{C}$, then $\lambda(f)=\langle f\rangle \in I$. Letting $\phi \alpha$ be the image of $\lambda D$ in $C=(D \backslash S) / I, \phi: \mathbb{S} \rightarrow C$ is a well-defined homomorphism.

Theorem. Let $X, \mathcal{O}$ be a reduced subscheme of a projective space over a field. The length homomorphism $\phi: \mathbb{E} \rightarrow C$ is injective. The image of $\phi$ is the subgroup of locally principal elements of $C$, which is a subquotient of $D$. Furthermore $\phi: \mathbb{S} \rightarrow C$ is surjective if and only if $\mathcal{O}_{p}$ is factorial for all prime ideals $p$ of $\mathcal{O}$ which aie contained in no element of $\mathcal{C}$.

Proof. The usual argument follows ([4, Propositions, pp. 65, 66]).

Let $\supseteqq \backslash C$ be the subgroup of $\supseteqq$ of divisors $D$ such that $D_{p}=(1)_{p}$ for all $p \in \mathcal{C}$. Let $D \in \mathfrak{D} \backslash \mathcal{C}$ with $\lambda D=\langle 1\rangle$. Let $p$ be a prime ideal of $\mathcal{O}$, and let $f_{1}$ and $f_{2}$ be regular elements of $\Theta_{p}$ such that $D_{p}=\left(f_{1} / f_{2}\right)_{p}$. If $q \subset p$ is a prime ideal contained in $\mathcal{C}$, then $D_{q}=(1)_{q}$ and $\mathcal{O}_{q} f_{1}=\mathcal{O}_{q} f_{2}$. If $q \subset p$ is a divisorial prime ideal not contained in $\mathcal{C}$, then $\ell_{\mathcal{O}_{q}}\left(\mathcal{O}_{q} / \mathcal{O}_{q} f_{1}\right)=\ell_{\mathcal{O}_{q}}\left(\mathcal{O}_{q} / \mathcal{O}_{q} f_{2}\right)$ and, because $\mathcal{O}_{q}$ is normal of Krull dimension one, $\mathcal{O}_{q} f_{1}=\mathcal{O}_{q} f_{2}$. Thus $\mathcal{O}_{q} f_{1}=$ $\mathcal{O}_{q} f_{2}$ for all depth one (grade one) prime ideals $q$ of $\mathcal{O}_{p}$, and

$$
\Theta_{p} f_{1}=\bigcap_{q} \Theta_{q} f_{1}=\bigcap_{q} \Theta_{q} f_{2}=\Theta_{p} f_{2} .
$$

Hence $\mathcal{O}_{p} f_{1}=\mathcal{O}_{p} f_{2}$ for all prime ideals $p$ of $\mathcal{O}, f_{1} / f_{2} \in \mathcal{O}_{p}^{*}, D=(1)$, and $\lambda$ : D) $\backslash \mathcal{C} \rightarrow D \backslash S$ is injective.

Now, to show that $\phi: \sqrt{S} \rightarrow C$ is injective, let $\alpha \in \mathbb{S}$ with $\phi \alpha=0 \in C$, and let $D \in \alpha$ be such that $D_{q}=(1)_{q}$ for all $q$ in $\mathcal{C}$. Let $f \in K^{*}$ be such that $f \in \mathcal{O}_{q}^{*}$ for all $q$ in $\mathcal{C}$ and $\lambda D=\langle f\rangle$. Then by the injectivity of $\lambda$ above, $D=$ $(f)$, and $\alpha=0$.

$\phi:(5) C$ is surjective if and only if $\lambda: D \backslash C \rightarrow D \backslash S$ is surjective. This is true if and only if the group of Cartier divisors is equal to the group 
of Weil divisors for each local ring $\Theta_{p}$ where $p$ is a prime ideal of $\mathcal{O}_{\text {which }}$ is contained in no element of $\mathcal{C}$, which is in turn equivalent to each $\mathcal{O}_{p}$ being factorial for all these prime ideals $p$. Q.E.D.

If $\mathcal{O}$ is not reduced, $\mathcal{C}$ may no longer be finite, and the construction used to define $\phi$ may not be applicable. For example, let $R=k[x, y, z] /\left(x^{2}, x y\right)$ where $k$ is a field. The reduction of $R, R / R x=k[y, z]$, is the polynomial ring in two variables over $k$. Let $p$ be a prime element of $k[z]$. $R p$ has as associated prime ideals $(p, x)$ and $(p, x, y)$, for $(x, y)$ is an embedded component of $(0)$ in

$$
(k[z] /(p))[x, y] /\left(x^{2}, x y\right) \simeq R / R p .
$$

Thus $(p, x, y) \in \mathscr{D}$, and $\mathscr{D}$ is infinite. The similar projective example given by the homogeneous ring $k[W, X, Y, Z] /\left(X^{2}, X Y\right)$ is such that every homogeneous height one prime ideal is contained in an element of $\mathscr{D}$.

\section{REFERENCES}

1. Séminaire C. Chevalley, 3ième année: $1958 / 59$, Variétés de Picard, Ecole Normal Supérieure, Paris, 1960. MR 28 \#1094.

2. J. Hornell, Divisorial complete intersections, Pacific J. Math. 45 (1973), 217-227. MR 47 \#6668.

3. S. Lang, Abelian varieties, Interscience Tracts in Pure and Appl. Math., no. 7, Interscience, New York, 1959. MR 21 \#4959.

4. D. Mumford, Lectures on curves on an algebraic surface, Ann. of Math. Studies, no. 59, Princeton Univ. Press, Princeton, N. J., 1966. MR 35 \#187.

5. M. Rosenlicht, Equivalence relations on algebraic curves, Ann. of Math. (2) 56 (1952), 169-191. MR 14, 80.

DEPARTMENT OF MATHEMATICS, UNIVERSITY OF KANSAS, LAWRENCE, KANSAS 66044

Current address: 2017 North 6th Street Terrace, Blue Springs, Missouri 64015 